\title{
Continuous symmetries and conservation laws in chiral media
}

Crimin, Frances, Mackinnon, Neel, Götte, Jörg, Barnett, Stephen

Frances Crimin, Neel Mackinnon, Jörg Götte, Stephen M. Barnett, "Continuous symmetries and conservation laws in chiral media," Proc. SPIE 11297, Complex Light and Optical Forces XIV, 112970J (24 February 2020); doi: $10.1117 / 12.2550815$

SPE. Event: SPIE OPTO, 2020, San Francisco, California, United States 


\title{
Continuous symmetries and conservation laws in chiral media
}

\author{
Frances Crimin $^{\mathrm{a}}$, Neel Mackinnon ${ }^{\mathrm{a}}$, Jörg B. Götte ${ }^{\mathrm{a}, \mathrm{b}}$, and Stephen M. Barnett ${ }^{\mathrm{a}}$ \\ ${ }^{a}$ Department of Physics and Astronomy, University of Glasgow, Glasgow G12 8QQ, UK \\ ${ }^{\mathrm{b}}$ College of Engineering and Applied Science, Nanjing University, Nanjing 210093, China
}

\begin{abstract}
Locally conserved quantities of the electromagnetic field in lossless chiral media are derived from Noether's theorem, including helicity, chirality, momentum, and angular momentum, as well as the separate spin and orbital components of this last quantity. We discuss sources and sinks of each in the presence of current densities within the material, and in some cases, as also generated by inhomogeneity of the medium. A previously obtained result connecting sources of helicity and energy within chiral materials is explored, revealing that association between the two quantities is not restricted to chiral media alone. Rather, it is analogous to the connection between the momentum, and the spin and orbital components of the total angular momentum. The analysis reveals a new quantity, appearing as the "orbital" counterpart of the helicity density in classical electromagnetism.
\end{abstract}

Keywords: Chiral media, conserved quantities, Noether's theorem, optical angular momentum, gauge transformations

\section{INTRODUCTION}

The propagation of electromagnetic fields in chiral media has long been a topic of interest in classical electromagnetism. $^{1-4}$ In the simplest case, a chiral medium is a macroscopic, continuous material made up of indistinguishable chiral objects that are distributed homogeneously throughout, but with random orientation. A chiral object is one which cannot be superimposed upon its mirror image by rotation or translation: such objects exhibit handedness. When a linearly polarised field passes through a chiral medium, the left- and right-circularly polarised components of the field travel at different group velocities, ${ }^{4,5}$ so that, upon leaving the medium and recombining, the polarisation of the transmitted field is rotated with respect to the incident one. ${ }^{5-8}$ The angle, $\theta$, through which this polarisation is rotated, differs in sign depending upon whether the constituent objects in the medium are left- or right-handed. In this way, chiral matter demonstrates handedness.

The simplest example of a chiral object is a helix, a geometric figure which is also traced by the electric and magnetic field vector of circularly polarised light. There has been a recent resurgence of activity in the study of the chiral content of light, ${ }^{9-13}$ and the optical helicity ${ }^{11,14-20}$ has been advocated as an appropriate measure of this. ${ }^{11,13,21}$ It has been shown ${ }^{22}$ that the helicity density, in particular, takes on a peculiar form in chiral media, acquiring an additional term which is proportional to the energy density, with a sign dependent upon the handedness of the material: sources of energy therefore become equivalent to sources of helicity in such media. This was previously observed by Lakhtakia et al., ${ }^{23}$ who found that the far-field radiation emitted by a point electric dipole in a chiral sphere is identical to that of an electric-magnetic dipole pair radiating into vacuum, which itself is a simple model of a source of helicity. ${ }^{21,24}$ The relationship between the helicity and the energy thus warrants further investigation, and so this curious result prompts us to consider whether such a relationship holds between other conserved quantities in chiral media, particularly those naturally associated with the angular momentum content of the field, in the hope that such findings may shed some light upon it.

In this article, we derive the form of some locally conserved quantities in chiral media from Noether's theorem, including the helicity, chirality, momentum and (spin and orbital) angular momentum densities. The forms of sources of these quantities in the presence of a local current are found, and the results discussed. Throughout, we consider only homogeneous, isotropic, and lossless media, unless otherwise stated.

Further author information: (Send correspondence to F.C.)

F.C.: E-mail: frances.crimin@glasgow.ac.uk

Complex Light and Optical Forces XIV, edited by David L. Andrews, Enrique J. Galvez,

Halina Rubinsztein-Dunlop, Proc. of SPIE Vol. 11297, 112970J · @ 2020 SPIE

CCC code: $0277-786 \mathrm{X} / 20 / \$ 21 \cdot$ doi: $10.1117 / 12.2550815$

Proc. of SPIE Vol. 11297 112970J-1 


\section{THE ENERGY DENSITY AND LAGRANGIAN DENSITY IN CHIRAL MEDIA}

In this section, we use the known form of the energy density in chiral media ${ }^{25,26}$ to postulate the form of the Lagrangian density, and then go on test this quantity by various means. The energy density in a chiral medium can be written in the exact form ${ }^{25,26}$

$$
w_{\beta}=\frac{1}{2}\left(\frac{1}{\epsilon} \mathbf{D} \cdot \mathbf{D}+\frac{1}{\mu} \mathbf{B} \cdot \mathbf{B}\right) .
$$

We use the Drude-Born-Fedorov (DBF) constitutive relations, ${ }^{27}$ where the electric and magnetic inductions $\mathbf{D}$ and $\mathbf{B}$ have the form

$$
\begin{aligned}
& \mathbf{D}=\epsilon(\mathbf{E}+\beta \boldsymbol{\nabla} \times \mathbf{E}), \\
& \mathbf{B}=\mu(\mathbf{H}+\beta \boldsymbol{\nabla} \times \mathbf{H}) .
\end{aligned}
$$

Here, the pseudoscalar $\beta$ describes the chiroptical response of the medium, with $\epsilon$ and $\mu$ acting as the permittivity and permeability. Using (2), it is straightforward to check the local conservation of the energy density (1). In the presence of a current density $\mathbf{j}$, we obtain

$$
\partial_{t} w_{\beta}+\nabla \cdot(\mathbf{E} \times \mathbf{H})=-\frac{1}{\epsilon} \mathbf{j} \cdot \mathbf{D} .
$$

Expansion of the energy density (1) using (2) highlights its peculiar form in a chiral medium: for each of the electric and magnetic contributions, it contains two terms which do not depend on the handedness of the material, and one, $\mathbf{E} \cdot(\boldsymbol{\nabla} \times \mathbf{E})$ and $\mathbf{H} \cdot(\boldsymbol{\nabla} \times \mathbf{H})$, which does, as it is proportional to $\beta$. This structure, the dot product of a field with its curl, is echoed by the form of both the optical chirality density, ${ }^{28}$ which in vacuum is given by

$$
\chi=\frac{\epsilon_{0}}{2}\left[\mathbf{E} \cdot(\boldsymbol{\nabla} \times \mathbf{E})+c^{2} \mathbf{B} \cdot(\boldsymbol{\nabla} \times \mathbf{B})\right],
$$

and the helicity density in vacuum ${ }^{11,18}$

$$
h=\frac{1}{2}\left[\sqrt{\frac{\epsilon_{0}}{\mu_{0}}} \mathbf{A}^{\perp} \cdot(\boldsymbol{\nabla} \times \mathbf{A})+\sqrt{\frac{\mu_{0}}{\epsilon_{0}}} \mathbf{C}^{\perp} \cdot(\boldsymbol{\nabla} \times \mathbf{C})\right],
$$

where the transverse part of $\mathbf{C}$ defines the transverse part of the displacement field, $\mathbf{D}^{\perp}=-\boldsymbol{\nabla} \times \mathbf{C} .^{29,30}$ This already hints at the connection between measures of the chiral content of a field and the energy density, ${ }^{22}$ which only becomes apparent if the medium is chiral.

From (1), we introduce the following form of the Lagrangian density of the free field in a chiral medium:

$$
\mathcal{L}=\frac{1}{2}\left(\frac{1}{\epsilon} \mathbf{D} \cdot \mathbf{D}-\frac{1}{\mu} \mathbf{B} \cdot \mathbf{B}\right) .
$$

Again using the DBF constitutive relations, this is written in terms of the vector and scalar coordinates of the electromagnetic potential, $\mathbf{A}$ and $A_{0}$, in an arbitrary gauge:

$$
\mathcal{L}=\frac{1}{2}\left(\epsilon\left(\dot{\mathbf{A}}+\nabla A_{0}+\beta \boldsymbol{\nabla} \times \dot{\mathbf{A}}\right)^{2}-\frac{1}{\mu}(\boldsymbol{\nabla} \times \mathbf{A})^{2}\right) .
$$

The momentum conjugate to $\mathbf{A}$ is

$$
\boldsymbol{\Pi}=\frac{\partial \mathcal{L}}{\partial \dot{\mathbf{A}}}=-(1+\beta \boldsymbol{\nabla} \times) \mathbf{D},
$$

and the form of (6) may be verified by the resulting Euler-Lagrange equations, since

$$
\frac{d}{d t}\left(\frac{\partial \mathcal{L}}{\partial \dot{\mathbf{A}}}\right)=-(1+\beta \nabla \times) \dot{\mathbf{D}}
$$


and

$$
\frac{\partial \mathcal{L}}{\partial \mathbf{A}}=-(1+\beta \boldsymbol{\nabla} \times) \boldsymbol{\nabla} \times \mathbf{H}
$$

such that the Maxwell equation $\dot{\mathbf{D}}=\boldsymbol{\nabla} \times \mathbf{H}$ is recovered. It is worthwhile to note that the Lagrangian density (6) can also be written in terms of the electric scalar and vector potentials, $C_{0}$ and $\mathbf{C}$. Following the above treatment for the generalised coordinate $\mathbf{A}$, the Euler-Lagrange equations in the present case reveal Faraday's law, $\dot{\mathbf{B}}=-\boldsymbol{\nabla} \times \mathbf{E}$. As a further test of (6), we find that the energy density (1) can be recovered by finding $\mathcal{H} \equiv w_{\beta}=\Pi \dot{\mathbf{A}}-\mathcal{L}$. Analogous results follow using the coordinate $\mathbf{C}$ and associated momentum.

\section{CONSERVED QUANTITIES}

We wish to test whether various electromagnetic quantities, conserved in vacuum, are also conserved in chiral media, and to find the explicit form of these quantities. To this end, we apply the infinitesimal transformation known to generate each conserved quantitiy in vacuum to the Lagrangian density (6). Should it remain invariant, we then use Noether's theorem ${ }^{31}$ to find the form of these quantities in chiral media.

\subsection{The helicity density in a chiral medium}

It is now well-known that the duality transformation is generated by the conservation of helicity in vacuum, ${ }^{16,17}$ and in lossless media in which dual-symmetry is preserved. ${ }^{19,22}$ The form of the helicity density in chiral media has been found previously, ${ }^{21,22}$ by considering its continuity equation directly. Here, we verify these results, and indeed the form of the Lagrangian density (6), by deriving it instead from Noether's theorem. We proceed by applying the infinitesimal form of the duality transformation ${ }^{32}$

$$
\begin{aligned}
& \mathbf{A} \rightarrow \mathbf{A}+\theta \sqrt{\frac{\mu}{\epsilon}} \mathbf{C}, \\
& \mathbf{C} \rightarrow \mathbf{C}-\theta \sqrt{\frac{\epsilon}{\mu}} \mathbf{A},
\end{aligned}
$$

to (6). Verifying that the Euler-Lagrange equations still hold upon this transformation, it is straightforward to find:

$$
\left(\frac{\partial \mathcal{L}}{\partial \dot{\mathbf{A}}}\right) \delta \mathbf{A}+\left(\frac{\partial \mathcal{L}}{\partial \dot{\mathbf{C}}}\right) \delta \mathbf{C}=\theta\left[-\sqrt{\frac{\mu}{\epsilon}} \mathbf{C} \cdot(1+\beta \boldsymbol{\nabla} \times) \mathbf{D}+\sqrt{\frac{\epsilon}{\mu}} \mathbf{A} \cdot(1+\beta \nabla \times) \mathbf{B}\right]
$$

such that the conserved quantity is given in the square brackets. After integration by parts, setting

$$
\int \nabla_{i}\left[\sqrt{\frac{\epsilon}{\mu}}(\mathbf{A} \times \mathbf{B})_{i}-\sqrt{\frac{\mu}{\epsilon}}(\mathbf{C} \times \mathbf{D})_{i}\right] d^{3} \mathbf{r} \equiv 0,
$$

and dividing the whole quantity by a factor of two, the helicity density in a chiral medium is identified:

$$
\begin{aligned}
h_{\beta} & =\frac{1}{2}\left[\sqrt{\frac{\epsilon}{\mu}} \mathbf{B} \cdot(1+\beta \boldsymbol{\nabla} \times) \mathbf{A}^{\perp}-\sqrt{\frac{\mu}{\epsilon}} \mathbf{D} \cdot(1+\beta \boldsymbol{\nabla} \times) \mathbf{C}^{\perp}\right] \\
& =\frac{1}{2}\left(\sqrt{\frac{\epsilon}{\mu}} \mathbf{A}^{\perp} \cdot \mathbf{B}-\sqrt{\frac{\mu}{\epsilon}} \mathbf{C}^{\perp} \cdot \mathbf{D}\right)+\beta \sqrt{\epsilon \mu} \frac{1}{2}\left(\frac{1}{\mu} \mathbf{B} \cdot \mathbf{B}+\frac{1}{\epsilon} \mathbf{D} \cdot \mathbf{D}\right) .
\end{aligned}
$$

We have further specified that only the transverse parts of the vector potentials are included in (14): this enables the helicity density to be written in a manifestly gauge-independent form, ${ }^{16}$ and indeed, these are the only parts of the vector potentials picked out by an integration over all space in calculating the total helicity. Eq. (14) is in agreement with the form of the helicity density in a chiral medium found in earlier work. ${ }^{22}$ It is worthwhile to

examine this equation in the presence of a current density $\mathbf{j}$ within the medium. Using the helicity flux density

$$
\mathbf{v}=\frac{1}{2}\left(\sqrt{\frac{\epsilon}{\mu}} \mathbf{E} \times \mathbf{A}^{\perp}+\sqrt{\frac{\mu}{\epsilon}} \mathbf{H} \times \mathbf{C}^{\perp}\right)
$$


and defining $\mathbf{j}^{\perp}=\boldsymbol{\nabla} \times \mathbf{g},{ }^{18}$ the continuity equation for the helicity density produces

$$
\partial_{t} h_{\beta}+\nabla \cdot \mathbf{v}=\frac{1}{2} \sqrt{\frac{\mu}{\epsilon}}[\mathbf{g} \cdot(\boldsymbol{\nabla} \times \mathbf{C})+\mathbf{C} \cdot(\boldsymbol{\nabla} \times \mathbf{g})]-\sqrt{\frac{\mu}{\epsilon}} \beta \mathbf{j}^{\perp} \cdot \mathbf{D} .
$$

This is exact to all $\mathcal{O}(\beta)$, i.e. there are no higher order source terms as previously expected. ${ }^{21}$ This result is comparable to the analogous continuity equation for the energy density, as shown in (3).

\subsection{Chirality density in a chiral medium}

Following the above treatment, we proceed to find the form of other well-known quantities of the electromagnetic field in a chiral medium, beginning with the chirality density. We consider the following infinitesimal transformation of the magnetic vector potentials $\mathbf{A}$ and $\mathbf{C}$, known to be genrated by the conservation of chirality in vacuum: ${ }^{33}$

$$
\begin{aligned}
& \mathbf{A} \rightarrow \mathbf{A}+\eta \boldsymbol{\nabla} \times \dot{\mathbf{A}}, \\
& \mathbf{C} \rightarrow \mathbf{C}+\eta \boldsymbol{\nabla} \times \dot{\mathbf{C}} .
\end{aligned}
$$

It is straightforward to check by substitution into the macroscopic Maxwell equations that these constitute a symmetry transformation of the system only when the values of $\mu, \epsilon$, and $\beta$ are constant. We would therefore expect chirality, and not helicity, ${ }^{19}$ to be generated at the interface between vacuum and a dual-symmetric chiral medium where the ratio $\epsilon / \mu$ is constant, but the separate permittivity and permeability may vary. In analogy

with the treatment for the helicity density above, we identify, up to surface terms, the following conserved quantity as the chirality density:

$$
\chi_{\beta}=\frac{1}{2}\left[\frac{1}{\epsilon} \mathbf{D} \cdot(\boldsymbol{\nabla} \times \mathbf{D})+\frac{1}{\mu} \mathbf{B} \cdot(\boldsymbol{\nabla} \times \mathbf{B})\right] .
$$

We proceed to find an exact expression for the source terms on the right-hand side of the chirality continuity equation (up to surface terms) in the presence of a current density $\mathbf{j}$ :

$$
\partial_{t} \chi_{\beta}+\frac{1}{2} \boldsymbol{\nabla} \cdot[\mathbf{E} \times(\boldsymbol{\nabla} \times \mathbf{H})-\mathbf{H} \times(\boldsymbol{\nabla} \times \mathbf{E})]=-\frac{1}{2}[\mathbf{j} \cdot(\boldsymbol{\nabla} \times \mathbf{E})+\mathbf{E} \cdot(\boldsymbol{\nabla} \times \mathbf{j})]-\beta(\boldsymbol{\nabla} \times \mathbf{j}) \cdot(\boldsymbol{\nabla} \times \mathbf{E}) .
$$

Setting $\beta=0$ reduces this result to that obtained for achiral media, ${ }^{18,34}$ and it is clear that it parallels the result for the helicity density (16).

\subsection{The canonical and kinetic momentum density in a chiral medium}

The conservation of electromagnetic momentum is associated with symmetry under translation of the vector potentials: ${ }^{35}$

$$
\begin{aligned}
& \mathbf{A}(\mathbf{r}, t) \rightarrow \mathbf{A}(\mathbf{r}+\boldsymbol{\eta}, t), \\
& \mathbf{C}(\mathbf{r}, t) \rightarrow \mathbf{C}(\mathbf{r}+\boldsymbol{\eta}, t) .
\end{aligned}
$$

We Taylor expand to first order, to write

$$
\begin{aligned}
& \mathbf{A}(\mathbf{r}, t) \rightarrow \mathbf{A}(\mathbf{r}, t)+(\boldsymbol{\eta} \cdot \boldsymbol{\nabla}) \mathbf{A}(\mathbf{r}, t), \\
& \mathbf{C}(\mathbf{r}, t) \rightarrow \mathbf{C}(\mathbf{r}, t)+(\boldsymbol{\eta} \cdot \boldsymbol{\nabla}) \mathbf{C}(\mathbf{r}, t),
\end{aligned}
$$

which is equivalent to

$$
\begin{aligned}
& A_{i} \rightarrow A_{i}+\eta_{j} \nabla_{j} A_{i}, \\
& C_{i} \rightarrow C_{i}+\eta_{j} \nabla_{j} C_{i},
\end{aligned}
$$


where repeated indices are summed over. Adding a gradient $\nabla_{i} \chi$ to the translation, and choosing $\chi=-\eta_{j} A_{j}$, the variation of the vector potential $\mathbf{A}$ becomes gauge invariant: ${ }^{36}$

$$
\begin{aligned}
& \delta A_{i}=\eta_{j} \nabla_{j} A_{i}-\eta_{j} \nabla_{i} A_{j}, \\
& =\eta_{j}\left(\nabla_{j} A_{i}-\nabla_{i} A_{j}\right) \\
& \equiv \eta_{j} \mathcal{F}_{j i} .
\end{aligned}
$$

We identify $\mathcal{F}_{i j}$ as the electromagnetic field tensor. The corresponding variation of the vector potential $\mathbf{C}$ is

$$
\delta C_{i}=\eta_{j} \nabla_{j} C_{i}-\eta_{j} \nabla_{i} C_{j} .
$$

These variations are now equivalent to the transformation

$$
\begin{aligned}
& \mathbf{A} \rightarrow \mathbf{A}-\boldsymbol{\eta} \times(\boldsymbol{\nabla} \times \mathbf{A}), \\
& \mathbf{C} \rightarrow \mathbf{C}-\boldsymbol{\eta} \times(\boldsymbol{\nabla} \times \mathbf{C}),
\end{aligned}
$$

which are straightforward to identify as a symmetry transformation of the Lagrangian density (6), with the resultant conserved quantity

$$
\mathbf{p}_{\beta}=\mathbf{D} \times \mathbf{B}+\frac{1}{2} \beta[(\boldsymbol{\nabla} \times \mathbf{D}) \times \mathbf{B}+\mathbf{D} \times(\boldsymbol{\nabla} \times \mathbf{B})],
$$

the momentum density in a chiral medium. In the presence of charges and currents, we find

$$
\partial_{t} \mathbf{p}_{\beta}+\boldsymbol{\nabla} \cdot \overline{\mathbf{T}}=-\frac{1}{2}\left[\rho\left(\mathbf{E}+\frac{1}{\epsilon} \mathbf{D}\right)+2 \mathbf{j} \times \mathbf{B}+\beta[(\boldsymbol{\nabla} \times \mathbf{j}) \times \mathbf{B}+\mathbf{j} \times(\boldsymbol{\nabla} \times \mathbf{B})]\right],
$$

where the $T_{i j}^{t h}$ component of the tensor $\overline{\mathbf{T}}$ is given by

$$
T_{i j}=\frac{1}{2} \delta_{i j}(\mathbf{D} \cdot \mathbf{E}+\mathbf{B} \cdot \mathbf{H})-\frac{1}{2}\left(D_{i} E_{j}+E_{i} D_{j}+B_{i} H_{j}+H_{i} B_{j}\right) .
$$

We may test the form of the momentum density by inserting the the fields of left- and right-circularly polarised plane waves into (26) and the energy density (1), finding that it correctly produces a momentum of $\hbar k$ per photon. Similarly, in calculating the flux density components $T_{i j}$ for the plane waves, the momentum density is found to travel at the group velocity of the medium. ${ }^{22}$ The form of $\mathbf{p}_{\beta}$ seems to suggest a kinetic momentum density in a chiral medium of the form

$$
\left.\mathbf{p}_{k, \beta}=\frac{1}{c^{2}}\left(\mathbf{E} \times \mathbf{H}+\frac{1}{2} \beta[(\boldsymbol{\nabla} \times \mathbf{E}) \times \mathbf{H})+\mathbf{E} \times(\boldsymbol{\nabla} \times \mathbf{H})\right]\right)
$$

This can be motivated by considering the ratio of $\mathbf{p}_{\beta}$ to $\mathbf{p}_{k, \beta}$ for right- and left-handed circularly polarised plane waves travelling in the $\hat{z}$ direction, which is

$$
\frac{\left\langle\mathbf{p}_{\beta}^{ \pm} \cdot \hat{z}\right\rangle}{\left\langle\mathbf{p}_{k, \beta}^{ \pm} \cdot \hat{z}\right\rangle}=\left(n^{ \pm}\right)^{2}
$$

where

$$
n^{ \pm}=\frac{c k}{\omega}=\frac{\sqrt{\epsilon \mu}(1 \pm \beta k)}{\sqrt{\epsilon_{0} \mu_{0}}}
$$

with the superscripts + and - denoting the results for the two polarisations. This result, (30), is what we would expect for the ratio of canonical to kinetic momentum in a dielectric. ${ }^{37}$ 


\subsection{Angular momentum in a chiral medium}

Using the transformation generated by the conservation of total angular momentum, ${ }^{38}$ and following the method above, we find that the angular momentum in a chiral medium, up to surface terms, is given by

$$
\mathbf{J}_{\beta}=\int \mathbf{r} \times\left[\mathbf{D} \times \mathbf{B}+\beta \frac{1}{2}[(\boldsymbol{\nabla} \times \mathbf{D}) \times \mathbf{B}+\mathbf{D} \times(\boldsymbol{\nabla} \times \mathbf{B})]\right] d^{3} \mathbf{r} .
$$

As expected, this is equal to the cross product of the position vector with the linear momentum (26). Following the method of the separation of the angular momentum density into spin and orbital components, ${ }^{38}$ we re-write (32)

$$
\begin{aligned}
\mathbf{J}_{\beta}= & \frac{1}{2} \int\left[\mathbf{D} \times \mathbf{A}^{\perp}+D_{j}(\mathbf{r} \times \boldsymbol{\nabla}) A_{j}^{\perp}+\beta\left[(\boldsymbol{\nabla} \times \mathbf{D}) \times \mathbf{A}^{\perp}+(\boldsymbol{\nabla} \times \mathbf{D})_{j}(\mathbf{r} \times \boldsymbol{\nabla}) A_{j}^{\perp}\right]\right. \\
& \left.+\mathbf{B} \times \mathbf{C}^{\perp}+B_{j}(\mathbf{r} \times \boldsymbol{\nabla}) C_{j}^{\perp}+\beta\left[(\boldsymbol{\nabla} \times \mathbf{B}) \times \mathbf{C}^{\perp}+(\boldsymbol{\nabla} \times \mathbf{B})_{j}(\mathbf{r} \times \boldsymbol{\nabla}) C_{j}^{\perp}\right]\right] d^{3} \mathbf{r},
\end{aligned}
$$

where the surface terms have been dropped. This suggests a spin density of the form

$$
\mathbf{s}_{\beta}=\frac{1}{2}\left(\mathbf{D} \times \mathbf{A}^{\perp}+\mathbf{B} \times \mathbf{C}^{\perp}+\beta\left[(\boldsymbol{\nabla} \times \mathbf{D}) \times \mathbf{A}^{\perp}+(\boldsymbol{\nabla} \times \mathbf{B}) \times \mathbf{C}^{\perp}\right]\right),
$$

with the orbital angular momentum density correspondingly given by

$$
\mathbf{l}_{\beta}=\frac{1}{2} D_{j}(\mathbf{r} \times \boldsymbol{\nabla}) A_{j}^{\perp}+B_{j}(\mathbf{r} \times \boldsymbol{\nabla}) C_{j}^{\perp}+\beta\left[(\boldsymbol{\nabla} \times \mathbf{D})_{j}(\mathbf{r} \times \boldsymbol{\nabla}) A_{j}^{\perp}+(\boldsymbol{\nabla} \times \mathbf{B})_{j}(\mathbf{r} \times \nabla) C_{j}^{\perp}\right] .
$$

The form of the spin and orbital components, (34) and (35), are in agreement with the results obtained using Noerther's theorem and the infinitesimal symmetry transformations generated by the conservation of the respective components. ${ }^{38}$

Let us examine the $\beta$-dependent terms in the spin density (34). Integrating by parts, we find

$$
\left.\int \beta\left[(\boldsymbol{\nabla} \times \mathbf{D}) \times \mathbf{A}^{\perp}+(\boldsymbol{\nabla} \times \mathbf{B}) \times \mathbf{C}^{\perp}\right)\right] d^{3} \mathbf{r}=2 \beta \int \mathbf{D} \times \mathbf{B} d^{3} \mathbf{r},
$$

so that the total spin density becomes

$$
\begin{aligned}
\mathbf{s}_{\beta} & =\frac{1}{2}\left(\mathbf{D} \times \mathbf{A}^{\perp}+\mathbf{B} \times \mathbf{C}^{\perp}\right)+\beta \mathbf{D} \times \mathbf{B} \\
& \equiv \mathbf{s}+\beta \mathbf{p},
\end{aligned}
$$

where $\mathbf{s}$ and $\mathbf{p}$ have the same form as the spin and linear momentum densities in achiral media, respectively. This is reminiscent of the presence of the energy density in the chiral contribution to the helicity density (14), although there, it is the full energy density which is included, whereas here we do not include the $\beta$ dependent term in $\mathbf{p}_{\beta}$ (26). The continuity equation of the spin density in the presence of sources reveals:

$$
\partial_{t} \mathbf{s}_{\beta}+\nabla \cdot \bar{\Sigma}=\frac{1}{2}\left[(\boldsymbol{\nabla} \times \mathbf{A}) \times \mathbf{g}+\mathbf{A}^{\perp} \times(\boldsymbol{\nabla} \times \mathbf{g})-2 \beta \mathbf{j}^{\perp} \times \mathbf{B}\right],
$$

where the $i j$-th component of the spin flux density $\bar{\Sigma}$ is defined as ${ }^{39}$

$$
\Sigma_{i j}=\frac{1}{2}\left[\delta_{i j}\left(\mathbf{A}^{\perp} \cdot \mathbf{H}-\mathbf{C}^{\perp} \cdot \mathbf{E}^{\perp}\right)-A_{i}^{\perp} H_{j}-H_{i} A_{j}^{\perp}+C_{i}^{\perp} E_{j}^{\perp}+E_{i}^{\perp} C_{j}^{\perp}\right] .
$$

Again in analogy with the helicity and energy relationship, by comparison to (27), we see the transverse components of sources of linear momentum contributing a net flux of spin density in a chiral medium. 
For completeness, the continuity equation for the orbital component of the angular momentum in the presence of currents and charges is given by

$$
\partial_{t} \mathbf{l}_{\beta}+\boldsymbol{\nabla} \cdot \overline{\boldsymbol{\Lambda}}=\frac{1}{2}\left[(\boldsymbol{\nabla} \times \mathbf{A})(\mathbf{r} \times \boldsymbol{\nabla}) \mathbf{g}-(\boldsymbol{\nabla} \times \mathbf{g})(\mathbf{r} \times \boldsymbol{\nabla}) \mathbf{A}^{\perp}+\beta\left[\mathbf{B}(\mathbf{r} \times \boldsymbol{\nabla}) \mathbf{j}^{\perp}-\mathbf{j}^{\perp}(\mathbf{r} \times \boldsymbol{\nabla}) \mathbf{B}\right]\right],
$$

where ${ }^{39}$

$$
\Lambda_{i j}=\frac{1}{2}\left[\varepsilon_{i k l} \varepsilon_{j m n} r_{k}\left[H_{n}\left(\nabla_{l} A_{m}^{\perp}\right)-E_{n}^{\perp}\left(\nabla_{l} C_{m}^{\perp}\right)\right]+H_{j} A_{i}^{\perp}-E_{j}^{\perp} C_{i}^{\perp}\right] .
$$

We note that in the absence of currents and charges, and in a non-dual-symmetric, achiral medium, the electric and magnetic contributions in the total, spin, and orbital angular momenta are separately conserved ${ }^{39}$ Inside a chiral medium, however, both electric and magnetic parts must be used together in order for conservation: dual symmetry is required for their conservation.

The forms of the energy, helicity, linear momentum, and spin densities, in addition to sources of each in the presence of a local current, are summarised in Table 1. The table highlights the connection between the conserved quantities in chiral media, which will be further discussed in the following section.

Table 1. Conserved quantities in chiral media, and sources of each in the presence of a current density $\mathbf{j}$ within the medium. The red highlight indicates the presence of the energy density, and its source term, in the form of the helicity density and its associated sources, with the blue indicating the analogous result for the linear momentum and spin density.

\begin{tabular}{|l|l|l|}
\hline Conserved quantity & Density & Sources \\
\hline Energy & $\frac{1}{2}\left(\frac{1}{\mu} \mathbf{B} \cdot \mathbf{B}+\frac{1}{\epsilon} \mathbf{D} \cdot \mathbf{D}\right)$ & $-\frac{1}{\epsilon} \mathbf{j} \cdot \mathbf{D}$ \\
\hline Helicity & $\frac{1}{2}\left(\sqrt{\frac{\epsilon}{\mu}} \mathbf{A}^{\perp} \cdot \mathbf{B}-\sqrt{\frac{\mu}{\epsilon}} \mathbf{C}^{\perp} \cdot \mathbf{D}\right)+\beta \frac{1}{c}\left(\frac{1}{\mu} \mathbf{B} \cdot \mathbf{B}+\frac{1}{\epsilon} \mathbf{D} \cdot \mathbf{D}\right)$ & $\frac{1}{2} \sqrt{\frac{\mu}{\epsilon}}\left(\mathbf{j}^{\perp} \cdot \mathbf{C}-\mathbf{g} \cdot \mathbf{D}-2 \beta \mathbf{j}^{\perp} \cdot \mathbf{D}\right)$ \\
\hline Linear momentum & $\mathbf{D} \times \mathbf{B}+\frac{1}{2} \beta((\boldsymbol{\nabla} \times \mathbf{D}) \times \mathbf{B}+\mathbf{D} \times(\boldsymbol{\nabla} \times \mathbf{B}))$ & $-\frac{1}{2}\left[\rho\left(\mathbf{E}+\frac{1}{\epsilon} \mathbf{D}\right)+2 \mathbf{j}^{\perp} \times \mathbf{B}+\beta\left(\left(\boldsymbol{\nabla} \times \mathbf{j}^{\perp}\right) \times \mathbf{B}+\mathbf{j}^{\perp} \times(\boldsymbol{\nabla} \times \mathbf{B})\right)\right]$ \\
\hline Spin & $\frac{1}{2}\left(\mathbf{D} \times \mathbf{A}^{\perp}+\mathbf{B} \times \mathbf{C}^{\perp}\right)+\beta \mathbf{D} \times \mathbf{B}$ & $\frac{1}{2}\left(\left((\boldsymbol{\nabla} \times \mathbf{A}) \times \mathbf{g}+\mathbf{A}^{\perp} \times\left((\boldsymbol{\nabla} \times \mathbf{g})-2 \beta \mathbf{j}^{\perp} \times \mathbf{B}\right)\right.\right.$ \\
\hline
\end{tabular}

\section{THE OPTICAL HELICITY AND ENERGY}

\subsection{The "orbital counterpart" of the helicity density}

The spin angular momentum can be derived from the total angular momentum, itself the cross product of the position vector with the linear momentum, and so we propose that the helicity, too, can be constructed from some product of the energy density with the position vector. It turns out there are at least two different methods for doing this, but we shall present the one which most closely resembles the derivation of the spin and orbital angular momentum from the total linear momentum. For simplicity in this section, we will consider fields in vacuum, rather than in chiral media, unless otherwise stated.

From the form of the energy density (1), we construct the quantity

$$
\frac{1}{2} \int\left(\frac{1}{\epsilon_{0}} \mathbf{D} \cdot(\mathbf{r} \times \mathbf{D})+\frac{1}{\mu_{0}} \mathbf{B} \cdot(\mathbf{r} \times \mathbf{B})\right) d^{3} \mathbf{r} \equiv 0,
$$

where the integrand itself is identically zero, as $\mathbf{B} \cdot(\mathbf{r} \times \mathbf{B})=\mathbf{r} \cdot(\mathbf{B} \times \mathbf{B})=0$, and similarly for the electric term. Focusing on the magnetic half only, we write $\mathbf{B}=\boldsymbol{\nabla} \times \mathbf{A}^{\perp}$ to produce

$$
\int \mathbf{B} \cdot(\mathbf{r} \times \mathbf{B}) d^{3} \mathbf{r}=\int\left(B_{i} r_{j} \nabla_{i} A_{j}^{\perp}-B_{i} r_{j} \nabla_{j} A_{i}^{\perp}\right) d^{3} \mathbf{r} .
$$

The first term on the right-hand side of (43) is integrated by parts, so that the total integral becomes

$$
0=-\int\left(\mathbf{A}^{\perp} \cdot \mathbf{B}+B_{i}(\mathbf{r} \cdot \nabla) A_{i}^{\perp}\right) d^{3} \mathbf{r}+\int \nabla_{i}\left(B_{i}\left(\mathbf{r} \cdot \mathbf{A}^{\perp}\right)\right) d^{3} \mathbf{r} .
$$


Applying the same treatment to the electric term $\mathbf{D} \cdot(\mathbf{r} \times \mathbf{D})$, we rewrite $(42)$

$$
\begin{aligned}
0= & \frac{1}{2} \int\left(\frac{1}{\mu_{0}} \mathbf{A}^{\perp} \cdot \mathbf{B}-\frac{1}{\epsilon_{0}} \mathbf{C}^{\perp} \cdot \mathbf{D}\right) d^{3} \mathbf{r}+\frac{1}{2} \int\left(\frac{1}{\mu_{0}} B_{i}(\mathbf{r} \cdot \nabla) A_{i}^{\perp}-\frac{1}{\epsilon_{0}} D_{i}(\mathbf{r} \cdot \nabla) C_{i}^{\perp}\right) d^{3} \mathbf{r} \\
& -\frac{1}{2} \int\left(\frac{1}{\mu_{0}} \nabla_{i}\left(B_{i}\left(\mathbf{r} \cdot \mathbf{A}^{\perp}\right)\right)-\frac{1}{\epsilon_{0}} \nabla_{i}\left(D_{i}\left(\mathbf{r} \cdot \mathbf{C}^{\perp}\right)\right)\right) d^{3} \mathbf{r} .
\end{aligned}
$$

The first integrand in (45) is straightforward to identify: multiplying it by the speed of light, we obtain exactly the helicity density in vacuum (5). Accordingly, we multiply the second integrand in (45) by $1 / \sqrt{\epsilon_{0} \mu_{0}}$ to define

$$
h_{o}=\frac{1}{2}\left(\sqrt{\frac{\epsilon_{0}}{\mu_{0}}} B_{i}(\mathbf{r} \cdot \nabla) A_{i}^{\perp}-\sqrt{\frac{\mu_{0}}{\epsilon_{0}}} D_{i}(\mathbf{r} \cdot \nabla) C_{i}^{\perp}\right),
$$

a quantity with units of a helicity density which seems to play the role of the "orbital counterpart" of this quantity. The final integral of (45) contains the surface terms left over from the integration by parts.

\subsection{Conservation of $h_{o}$}

As is well known, the spin density also plays the role of the helicity flux density: ${ }^{11}$ the exact relationship is

$$
\mathbf{s}=\frac{\mathbf{v}}{c}
$$

where $\mathbf{v}$ is the helicity flux density, and $c$ is the speed of light in vacuum. It would be reasonable to expect, therefore, that the orbital angular momentum density also plays the role of the flux of $h_{o}$. After some calculation, indeed we find

$$
\partial_{t} h_{o}+c \boldsymbol{\nabla} \cdot \mathbf{l}=\frac{1}{2} \sqrt{\frac{\mu_{0}}{\epsilon_{0}}}\left[(\boldsymbol{\nabla} \times \mathbf{g})_{i}(\mathbf{r} \cdot \nabla) C_{i}^{\perp}+(\boldsymbol{\nabla} \times \mathbf{C})_{i}(\mathbf{r} \cdot \boldsymbol{\nabla}) g_{i}\right],
$$

where the right-hand side reduces to zero in the absence of sources, and $\mathbf{l}$ is the orbital angular momentum density in vacuum, given by setting $\beta=0$ in (35). The factor of $c$ follows from the relationship between the helicity flux density and the spin angular momentum density (47). The source terms on the right-hand side of (48) clearly have their counterpart in the source terms of helicity (for $\beta=0$ ), shown in (16).

The orbital angular momentum density is therefore equivalent to the flux density of $h_{o}$, with $h_{o}$ itself being a conserved quantity in the absence of currents. In contrast to both the energy density and the helicity density which require both the electric and magnetic halves for conservation, ${ }^{21}$ the electric and magnetic halves of $h_{o}$ are separately conserved. Furthermore, it is found that the form of $h_{o}$ in chiral media remains unchanged from the vacuum definition, apart from the values of the permittivity and permeability, unlike the energy and helicity densities.

\subsection{Symmetries and conservation laws of $h_{o}$}

As $h_{o}$ is a locally conserved quantity, it must generate a symmetry transformation of the Lagrangian density (6). Let us rewrite the magnetic half of $h_{o}$, which follows from (44):

$$
\begin{aligned}
B_{i}(\mathbf{r} \cdot \nabla) A_{i}^{\perp} & =\mathbf{B} \cdot \nabla\left(\mathbf{r} \cdot \mathbf{A}^{\perp}\right)-\mathbf{B} \cdot \mathbf{A}^{\perp} \\
& =-\mathbf{B} \cdot\left(\mathbf{A}^{\perp}-\nabla\left(\mathbf{r} \cdot \mathbf{A}^{\perp}\right)\right) .
\end{aligned}
$$

The quantity in brackets on the right-hand side of (49) has the form of a gauge transformation of the magnetic vector potential. Similarly, for the electric half of $h_{o}$, we identify

$$
D_{i}(\mathbf{r} \cdot \boldsymbol{\nabla}) C_{i}^{\perp}=-\mathbf{D} \cdot\left(\mathbf{C}^{\perp}-\nabla\left(\mathbf{r} \cdot \mathbf{C}^{\perp}\right)\right) .
$$

The quantity $h_{o}$, whose flux density is given by the orbital angular momentum density, is therefore equivalent to a gauge transformation of the (negative) helicity density. From (43), we identify $B_{i} r_{j} \nabla_{j} A_{i}=B_{i} r_{j} \nabla_{i} A_{j}$, and 
similarly $D_{i} r_{j} \nabla_{j} C_{i}=D_{i} r_{j} \nabla_{i} C_{j}$. The symmetry transformations generated by the conservation of $B_{i} r_{j} \nabla_{i} A_{j}$ and $D_{i} r_{j} \nabla_{i} C_{j}$ (and therefore the magnetic and electric components of $h_{o}$ ) are found to be

$$
A_{i} \rightarrow A_{i}+\eta \sqrt{\frac{\mu}{\epsilon}}\left(C_{i}-\nabla_{i}(\mathbf{r} \cdot \mathbf{C})\right), \quad C_{i} \rightarrow C_{i}-\eta \sqrt{\frac{\epsilon}{\mu}}\left(A_{i}-\nabla_{i}(\mathbf{r} \cdot \mathbf{A})\right) .
$$

We recognise these as the symmetry transformation generated by the conservation of helicity, where the potentials $\mathbf{A}$ and $\mathbf{C}$ on the right-hand side are replaced by the gauge transformed quantities.

\subsection{Calculating $h_{o}$}

The surface terms resulting from the integration by parts in forming $h$ and $h_{o}$ from the energy density are given in the second line of (45), and can be rewritten

$$
\int\left(\frac{1}{\mu_{0}} \mathbf{B} \cdot \boldsymbol{\nabla}\left(\mathbf{r} \cdot \mathbf{A}^{\perp}\right)-\frac{1}{\epsilon_{0}} \mathbf{D} \cdot \boldsymbol{\nabla}\left(\mathbf{r} \cdot \mathbf{C}^{\perp}\right)\right) d^{3} \mathbf{r} .
$$

As the gradient functions are purely longitudinal, and $\mathbf{B}$ and $\mathbf{D}$ purely transverse, the integral over all space of each of the magnetic and electric contributions will be zero. ${ }^{40}$ From (45), it therefore follows that

$$
\int h_{o} d^{3} \mathbf{r}=-\int h d^{3} \mathbf{r}:
$$

the total helicity, and its orbital counterpart, are equal and opposite. Thus the integrated value our new quantity, $h_{o}$, seems to reveal no new information about the field. We note, however, that it is the flux of a quantity which characterises its flow across a surface per unit time, ${ }^{41}$ and corresponds to the measured observable. In this case, the flux of $h_{o}$, as shown in 4.2, has the form of the orbital angular momentum density of the field, whereas the flux of helicity is the spin density. ${ }^{11}$ The spin and orbital angular momentum components of light correspond to distinct quantities ${ }^{38}$ characterising different features of the field: the spin is determined by the polarisaton, whereas the orbital angular momentum measures the phase gradient of the waves. It is therefore worthwhile to examine whether these quantities are similarly related by the transformation of the vector potentials

$$
\begin{aligned}
& \mathbf{A} \rightarrow \mathbf{A}-\boldsymbol{\nabla}(\mathbf{r} \cdot \mathbf{A}), \\
& \mathbf{C} \rightarrow \mathbf{C}-\boldsymbol{\nabla}(\mathbf{r} \cdot \mathbf{C}) .
\end{aligned}
$$

For completeness, the corresponding transformation of the scalar potentials $A_{0}$ and $C_{0}$ is

$$
\begin{aligned}
& A_{0} \rightarrow A_{0}+\partial_{t}(\mathbf{r} \cdot \mathbf{A}), \\
& C_{0} \rightarrow C_{0}+\partial_{t}(\mathbf{r} \cdot \mathbf{C}),
\end{aligned}
$$

although these do not feature in the present transformation of the spin density, as only transverse components of the $\mathbf{A}$ and $\mathbf{C}$ fields are involved. Let us begin with the spin density in vacuum:

$$
\mathbf{s}=\frac{1}{2}\left(\mathbf{D} \times \mathbf{A}^{\perp}+\mathbf{B} \times \mathbf{C}^{\perp}\right) .
$$

Applying (54), we find:

$$
\begin{aligned}
\mathbf{s} & \rightarrow\left(\mathbf{D} \times \mathbf{A}^{\perp}+\mathbf{B} \times \mathbf{C}^{\perp}-\left(\mathbf{D} \times \boldsymbol{\nabla}\left(\mathbf{r} \cdot \mathbf{A}^{\perp}\right)+\mathbf{B} \times \boldsymbol{\nabla}\left(\mathbf{r} \cdot \mathbf{C}^{\perp}\right)\right)\right) \\
& =\left(\mathbf{D} \times \mathbf{A}^{\perp}+\mathbf{B} \times \mathbf{C}^{\perp}-\left(\mathbf{D} \times \mathbf{A}^{\perp}+\mathbf{r}(\mathbf{D} \cdot \mathbf{B})+D_{j}(\mathbf{r} \times \boldsymbol{\nabla}) A_{j}^{\perp}+\mathbf{B} \times \mathbf{C}^{\perp}-\mathbf{r}(\mathbf{B} \cdot \mathbf{D})+B_{j}(\mathbf{r} \times \boldsymbol{\nabla}) C_{j}^{\perp}\right)\right) \\
& =-\frac{1}{2}\left(D_{j}(\mathbf{r} \times \boldsymbol{\nabla}) A_{j}^{\perp}+B_{j}(\mathbf{r} \times \boldsymbol{\nabla}) C_{j}^{\perp}\right) \\
& =-\mathbf{l},
\end{aligned}
$$

where the last lines indeed reveal the negative orbital angular momentum density of the field. It therefore follows that the measurable components of the helicity density and its orbital counterpart, the spin and orbital angular 
momentum densities, are related by the gauge transformation (54). The fact that these fluxes are related by a gauge transformation is in accordance with the fact that $h_{o}$ integrated over all space measures exactly the helicity of the field: we would not expect a gauge transformed quantity to reveal anything other than the original quantity itself.

It is worthwhile here to again consider chiral media. Although the full orbital and spin angular momenta can be derived from the linear momentum density in chiral media (see 3.4), this is not the case for $h$ and $h_{o}$ found from the energy density, as seen in section 4.1. This is connected to the fact that only the achiral part of the linear momentum density appears in the chiral spin density, whereas the full energy density appears in the chiral from of the helicity density, as seen in Table 1. This has consequences, too, for the relationship between $h$ and $h_{o}$ in chiral media: they are no longer related by the gauge transformation (54). In turn, the spin and orbital angular momentum densities are also not related by (54) in a chiral medium.

The quantity $h_{o}$ arguably has a natural positioning amongst our various angular momentum measures. Indeed, in the same way that the helicity density, spin density, and spin flux density form a "helicity array", ${ }^{11}$ it would be natural to form such an array with $h_{o}$, the orbital angular momentum density, and its associated flux density. The meaning of $h_{o}$, however, remains ambiguous. As is clear from the above analysis, the quantity itself may be of no new significance in measuring the angular momentum content of the field. And yet, what it reveals about the connection between spin and orbital angular momentum components of light surely warrants further study. In addition, the relationship between these components in chiral media may shed further light upon the interaction of light with such matter.

\subsection{The gauge transformation}

Here we briefly examine the form of the gauge transformation (54). It is reminiscent of the transformation to the Power-Zienau-Wooley (PZW), or multipolar, gauge, familiar from electrodynamics. ${ }^{42-44}$ An arbitrary gauge transformation of the magnetic scalar and vector potentials is given by

$$
\begin{aligned}
\mathbf{A}(\mathbf{r}) \rightarrow \mathbf{A}\left(\mathbf{r}^{\prime}\right) & =\mathbf{A}(\mathbf{r})-\nabla \chi(\mathbf{r}), \\
A_{0}(\mathbf{r}) \rightarrow A_{0}\left(\mathbf{r}^{\prime}\right) & =A_{0}(\mathbf{r})+\dot{\chi}(\mathbf{r}),
\end{aligned}
$$

and the PZW gauge is obtained with the choice $\chi_{\mathrm{M}}(\mathbf{r}),{ }^{45}$

$$
\chi_{\mathrm{M}}(\mathbf{r})=-\int d \mathbf{r}^{\prime} \int_{0}^{1} d \lambda A_{j}\left(\mathbf{r}^{\prime}\right) r_{j} \delta\left(\mathbf{r}^{\prime}-\lambda \mathbf{r}\right) .
$$

For comparison, the gauge transformation in (54) is generated by the choice $\chi_{h}(\mathbf{r})=A_{j}(\mathbf{r}) r_{j}$. In the case of the PZW transformation, the new vector potential generated by (59) is ${ }^{45}$

$$
\mathbf{A}_{\mathrm{M}}(\mathbf{r})=\frac{1}{e} \int \boldsymbol{\theta}\left(\mathbf{r}^{\prime}, \mathbf{r}\right) \times \mathbf{B}\left(\mathbf{r}^{\prime}\right) d \mathbf{r}^{\prime}
$$

where

$$
\boldsymbol{\theta}\left(\mathbf{r}^{\prime}, \mathbf{r}\right)=-e \int_{0}^{1} d \lambda \lambda \mathbf{r} \delta\left(\mathbf{r}^{\prime}-\lambda \mathbf{r}\right)
$$

The vector potential defined by the gauge transformation (54) is

$$
\mathbf{A}_{h}(\mathbf{r})=-r_{j} \nabla_{i} A_{j}(\mathbf{r})
$$

It should be noted that the PZW gauge transformation is conventionally only applied to the magnetic potentials; its application and meaning in transforming the electric potentials $\mathbf{C}$ and $C_{0}$ has not, to the authors' knowledge, yet been discussed in the literature. The PZW gauge is commonly used for a system of charges and currents coupling with the fields, as it conveniently expresses this interaction as a function of the fields, rather than of the vector potentials. ${ }^{40}$ Furthermore, the polarisation and magnetisation densities, written as functions of microscopic observables, describe fully the system of charges, such that application to bulk media becomes 
straightforward. ${ }^{40}$ The similarity of the generator of the PZW gauge (59) to $\chi_{h}$, the generator which converts the spin to the orbital angular momentum density, is worthy of further investigation, and will be discussed in later work. As the PZW transformation is only effective in the case of interaction of a system of charges with the field, it will be pertinent to study the relationship between $\mathbf{s}$ and $\mathbf{l}$ in this case. Extension to linear media and then chiral matter will then follow as a matter of course.

\section{CONCLUSION}

In this article, we have presented the forms of a number of conserved quantities in chiral media, in an effort to study how measures of the chiral content of light change upon interaction with such matter. It has been shown that the helicity, chirality, momentum, and (spin and orbital) angular momentum are conserved in lossless chiral media, where the values of $\epsilon, \mu$ and $\beta$ are constant. The previously noted connection between the helicity and energy in a chiral medium has been further examined, and shown to be analogous to the connection between the spin and linear momentum inside the medium. This led to the recognition of the quantity $h_{o}$, which plays the role of the orbital counterpart of the helicity density, and whose flux is given by the orbital angular momentum density. It is shown to be related to the helicity by a straightforward gauge transformation, from which it follows that the same connection exists between the spin and orbital components of the angular momentum densities. Far from just being an interesting exercise in vector calculus, these findings may yet reveal some new insight into measuring the angular momentum content of light.

\section{ACKNOWLEDGMENTS}

This research was funded by The Royal Society under grant numbers RP/EA/180010 and RP/150122, and the Engineering and Physical Sciences Research Council under grant number EP/N509668/1. We would also like to acknowledge Niclas Westerberg, and thank him for useful discussions on the content of this paper.

\section{REFERENCES}

[1] Jaggard, D. L., Mickelson, A. R., and Papas, C. H., "On Electromagnetic Waves in Chiral Media," tech. rep. (1979).

[2] Lakhtakia, A., Varadan, V. V., and Varadan, V. K., "Field equations, Huygens's principle, integral equations, and theorems for radiation and scattering of electromagnetic waves in isotropic chiral media," J. Opt. Soc. Am. A 5, 175 (1988).

[3] Bassiri, S., Papas, C. H., and Engheta, N., "Electromagnetic wave propagation through a dielectric-chiral interface and through a chiral slab," J. Opt. Soc. Am. A 5, 1450 (1988).

[4] Lekner, J., "Optical properties of isotropic chiral media," Pure Appl. Opt. 5, 417 (1996).

[5] Biot, J.-B., "Phénomènes de polarisation successive, observés dans les fluides homogènes," Bull. Soc. Philomath 1, 190-192 (1815).

[6] Arago, D., "Sur une modification remarquable qu'éprouvent les rayons lumineux dans leur passage à travers certains corps diaphanes, et sur quelques autres nouveaux phénomènes d'optique," Mem. Inst. 1, 93-134 (1811).

[7] Pasteur, L., "Recherches sur les relations qui peuvent exister entre la forme cristalline, la composition chimique et le sens de la polarisation rotatoire," Ann. Chim. Phys 24, 442-459 (1848).

[8] Van'T Hoff, J. H., Pasteur, L., Richardson, G. M., and Le Bel, J. A., [The Foundations of Stereo Chemistry: Memoirs by Pasteur, Van'T Hoff, Lebel and Wilslincenus], American Book Company (1901).

[9] Bliokh, K. Y. and Nori, F., "Characterizing optical chirality," Phys. Rev. A - At. Mol. Opt. Phys. 83(2) (2011).

[10] Coles, M. M. and Andrews, D. L., "Chirality and angular momentum in optical radiation," Phys. Rev. A 85, 63810-7 (2012).

[11] Cameron, R. P., Barnett, S. M., and Yao, A. M., "Optical helicity, optical spin and related quantities in electromagnetic theory," New J. Phys. 14, 053050-16 (2012).

[12] Vázquez-Lozano, J. E. and Martínez, A., "Optical Chirality in Dispersive and Lossy Media," Phys. Rev. Lett. 121, 043901-7 (2018). 
[13] Mackinnon, N., "On the differences between helicity and chirality," J. Opt. 21, 125402 (2019).

[14] Calkin, M. G., "An Invariance Property of the Free Electromagnetic Field," Am. J. Phys. 33, 958-960 (1965).

[15] Trueba, J. L. and Rañada, A. F., "The electromagnetic helicity," Eur. J. Phys. 17, 141-144 (1996).

[16] Barnett, S. M., Cameron, R. P., and Yao, A. M., "Duplex symmetry and its relation to the conservation of optical helicity," Phys. Rev. A 86, 013845-4 (2012).

[17] Fernandez-Corbaton, I., Zambrana-Puyalto, X., Tischler, N., Vidal, X., Juan, M. L., and Molina-Terriza, G., "Electromagnetic duality symmetry and helicity conservation for the macroscopic maxwell's equations," Phys. Rev. Lett. 111, 060401-5 (2013).

[18] Nienhuis, G., "Conservation laws and symmetry transformations of the electromagnetic field with sources," Phys. Rev. A 93, 023840-9 (2016).

[19] van Kruining, K. and Götte, J. B., "The conditions for the preservation of duality symmetry in a linear medium," J. Opt 18, 085601-6 (2016).

[20] Alpeggiani, F., Bliokh, K. Y., Nori, F., and Kuipers, L., "Electromagnetic Helicity in Complex Media," Phys. Rev. Lett. 120, 243605-6 (2018).

[21] Crimin, F., Mackinnon, N., Götte, J. B., and Barnett, S. M., "Optical Helicity and Chirality: Conservation and Sources," Appl. Sci. 9, 828 (2019).

[22] Crimin, F., Mackinnon, N., Götte, J. B., and Barnett, S. M., "On the conservation of helicity in a chiral medium," J. Opt. 21, 094003 (2019).

[23] Lakhtakia, A., Varadan, V. K., and Varadan, V. V., "Radiation by a point electric dipole embedded in a chiral sphere," J. Phys. D App. Phys. 23, 481-485 (1990).

[24] Leeder, J. M., Haniewicz, H. T., and Andrews, D. L., "Point source generation of chiral fields: measures of near-and far-field optical helicity," J. Opt. Soc. Am. B 32, 2308-2313 (2015).

[25] Fedorov, F. I., [Terorija girotropii (Minsk: Nauka i Technika)] (1976).

[26] Proskurin, I., Ovchinnikov, A. S., Nosov, P., and Kishine, J. I., "Optical chirality in gyrotropic media: Symmetry approach," New J. Phys. 19, 063021 (2017).

[27] Lakhtakia, A., [Beltrami Fields in Chiral Media], vol. 2 of World Scientific Series in Contemporary Chemical Physics, World Scientific, Singapore (1994).

[28] Lipkin, D. M., "Existence of a new conservation law in electromagnetic theory," J. Math. Phys. 5, 696-700 (1964).

[29] Stratton, J. A., [Electromagnetic theory], McGraw-Hill Book Company (1941).

[30] Cameron, R. P., "On the 'second potential' in electrodynamics," J. Opt. 16, 015708-7 (2014).

[31] Noether, E., "Invariante Variationsprobleme," Nachrichten von der Gesellschaft der Wissenschaften zu Göttingen, Math. Klasse 1918, 235-257 (1918).

[32] Cameron, R. P. and Barnett, S. M., "Electric-magnetic symmetry and Noether's theorem," New J. Phys. 14, 123019 (2012).

[33] Philbin, T. G., "Lipkin's conservation law, Noether's theorem, and the relation to optical helicity," Phys. Rev. A - At. Mol. Opt. Phys. (4), 043843 (2013).

[34] Tang, Y. and Cohen, A. E., "Optical Chirality and Its Interaction with Matter," Phys. Rev. Lett. , 163901-4 (apr 2010).

[35] Philbin, T. G., "Electromagnetic energy momentum in dispersive media," Phys. Rev. A 83, 13823 (2011).

[36] Mieling, T. B., "Noether's theorem applied to classical electrodynamics," (4) (2017).

[37] Barnett, S. M. and Loudon, R., "The enigma of optical momentum in a medium," Philos. Trans. R. Soc. A Math. Phys. Eng. Sci. 368(1914), 927-939 (2010).

[38] Barnett, S. M., Allen, L., Cameron, R. P., Gilson, C. R., Padgett, M. J., Speirits, F. C., and Yao, A. M., "On the natures of the spin and orbital parts of optical angular momentum," J. Opt. 18, 064004-11 (2016).

[39] Bliokh, K. Y., Dressel, J., and Nori, F., "Conservation of the spin and orbital angular momenta in electromagnetism," New J. Phys. 16(9), 093037 (2014).

[40] Cohen-Tannoudji, C., Dupont-Roc, J., and Grynberg, G., [Photons and atoms: introduction to quantum electrodynamics], Wiley, New York (1989). 
[41] Barnett, S. M., "Optical angular-momentum flux," J. Opt. B Quantum Semiclassical Opt. 4, 7-16 (2002).

[42] Göppert-Mayer, M., "Über Elementarakte mit zwei Quantensprüngen," Ann. Phys. 9, 273-294 (1931).

[43] Power, E. A. and Zienau, S., "On the radiative contributions to the Van der Waals force," Nuovo Cim. 6, 7-17 (1957).

[44] Richards, P. I., "On the Hamiltonian for a particle in an electromagnetic field," Phys. Rev. 73, 254 (1948).

[45] Babiker, M. and Loudon, R., "Derivation of the Power-Zienau-Woolley Hamiltonian in Quantum Electrodynamics by Gauge Transformation," Proc. R. Soc. A Math. Phys. Eng. Sci. 385(9), 439-460 (1983). 